\title{
A Survey of Image Gradient Inversion Against Federated Learning
}

This paper was downloaded from TechRxiv (https://www.techrxiv.org).

LICENSE

CC BY 4.0

SUBMISSION DATE / POSTED DATE

$12-01-2022$ / 14-01-2022

CITATION

Li, Zhaohua; Wang, Le; Chen, Guangyao; Shafq, Muhammad; Gu, zhaoquan (2022): A Survey of Image Gradient Inversion Against Federated Learning. TechRxiv. Preprint.

https://doi.org/10.36227/techrxiv.18254723.v1

DOI

10.36227/techrxiv.18254723.v1 


\title{
A Survey of Image Gradient Inversion Against Federated Learning
}

\author{
Zhaohua Li, Le Wang*, Guangyao Chen, Muhammad Shafiq, and Zhaoquan Gu
}

\begin{abstract}
In order to preserve data privacy while fully utilizing data from different owners, federated learning is believed to be a promising approach in recent years. However, aiming at federated learning in the image domain, gradient inversion techniques can reconstruct the input images on pixel-level only by leaked gradients, without accessing the raw data, which makes federated learning vulnerable to the attacks. In this paper, we review the latest advances of image gradient inversion techniques and evaluate the impact of them to federated learning from the attack perspective. We use eight models and four datasets to evaluate the current gradient inversion techniques, comparing the attack performance as well as the time consumption. Furthermore, we shed light on some important and interesting directions of gradient inversion against federated learning.
\end{abstract}

Index Terms-Data privacy, federated learning, deep learning, gradient inversion.

\section{INTRODUCTION}

$\mathbf{N}$ OW deep learning has been used in many fields, especially in health care to improve medical diagnosis [1]. Constructing deep learning models often entails large quantities of data. For example, for a medical artificial intelligence (AI) model to develop into a professional medical expert, it needs to undergo sufficient training and have practical "experience" as a human physician does. On the other hand, the data required for model training are often owned by different institutions who do not share data, and collecting these data together may arouse data privacy issues or, worse still, legal concerns. Thus, federated learning (FL) [2] was proposed to ensure data privacy. Federated learning is a machine learning framework that allows multiple parties or multiple computing nodes to aggregate models without directly exchanging data. Federated learning has been intensively used in medical imaging for breast density classification [3], brain tumor segmentation [4], meta-analyses of large-scale subcortical brain data [5], prostate cancer classification [6], and oxygen demand prediction for COVID-19 patients [7], etc. In other words, federated learning leverages TOPS and data owned by

Date of current version January 5, 2022.

Z. Li, G. Chen, M. Shafiqi and Z. Gu are with Cyberspace Institute of Advanced Technology, Guangzhou University, Guangzhou, China (e-mail: \{lizhaohua, guangyaochen\}@e.gzhu.edu.cn; srsshafiq@gmail.com; zqgu@gzhu.edu.cn).

Corresponding author: L. Wang (e-mail: wanglelemail@yeah.net), Cyberspace Institute of Advanced Technology, Guangzhou University; and Peng Cheng Laboratory.

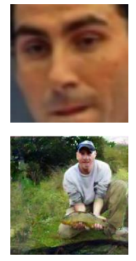

Target

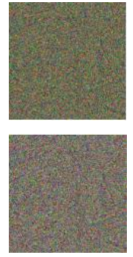

0

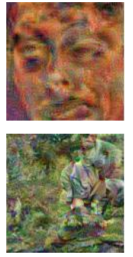

1000

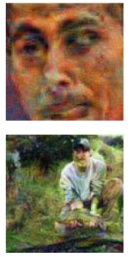

3000

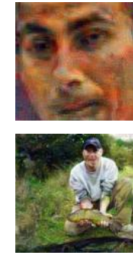

10000

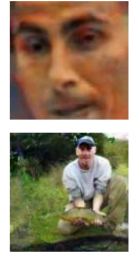

20000
Fig. 1. The process of Gradient Inversion reconstructing the input image. Row: the reconstructed sample, column: the number of iterations. The above images are from the datasets LFW [9], ImageNet [10].

multiple institutions without raw data sharing, which avoids revealing privacy and involved in the law.

However, Zhu et al. [8] found that a method, which utilizes only the gradient information leaked from federated learning model, could reconstruct original training data. This kind of method was later called the gradient inversion attack, it means that gradients exchanged among federated learning parties could result in privacy leakage. The gradient inversion technique studied in this paper is mainly for image data, which we refer to as Image Gradient Inversion. The input image reconstructed from the model gradient is shown in Fig. 1. The recovery is pixel-wise accurate for images, which means key features or sensitive information in raw data could be refactored. The privacy security of federal learning deserves careful study.

This paper provides an overview of the development and challenges of the gradient inversion technique concerning of federated learning privacy leakage. First, we review and clarify the gradient inversion paradigm and application scenarios. In federated learning with a parameter server, the identity of the data thief is not necessarily the parameter server; rather, the clients can also use gradient inversion to steal data. Second, we discuss how the literature on gradient inversion has evolved. Then, we compare several core methods of gradient inversion on different scale models by batch repeatable experiments, and reason the results and causes by theoretical analysis. Finally, we address important challenges and research directions for gradient inversion research.

The organization of this paper is as follows: Section II describes the relevant background of federated learning; Section III defines gradient inversion and outlines its applicable scenarios and components; Section IV reviews the development of gradient inversion, including an analysis of technological innovations in this regard; Section $\mathrm{V}$ elaborates on four key 
components of gradient inversion algorithms, and reveals how they affect the final result of the algorithm through experiments; In Section VI, we further analyze the opportunities to optimize existing gradient inversion methods, as well as the time consumption of gradient inversion on convolutional networks of different scales; The potential future prospects are discussed in Section VII; In Section VIII, we will explain other data leakage methods besides gradient inversion.

\section{FEDERATED LEARNING OVERVIEW}

\section{A. Federated Learning}

Federated learning was first proposed by McMahan et al.. [2], and the underlying idea of training models without collecting raw training data to a single location has proven to be useful in many practical scenarios. When data cannot be shared due to confidentiality concerns and legal restrictions, federated learning can be used to jointly model data knowledge from various institutions [11], [12].

In federated learning, the goal of building a global neural network is to minimize the objective function [13]

$$
\begin{array}{r}
\mathcal{L}(W)=\mathbb{E}_{i \sim p}\left[\mathcal{L}_{i}(W)\right] \\
\text { where } \quad \mathcal{L}_{i}(W)=\mathbb{E}_{\xi \sim \mathcal{D}_{i}}\left[\ell_{i}(W, \xi)\right]
\end{array}
$$

where $W \in \mathbb{R}^{d}$ represents the parameters of the global model, $\mathcal{L}_{i}: \mathbb{R}^{d} \rightarrow \mathbb{R}$ represents the local loss function of the $i^{t h}$ client, and $p$ represents the distribution of the overall client. Generally, all clients use the same local loss function $\ell_{i}$, but the data distribution owned by each client will be different.

\section{B. Model Aggregation}

In previous works on gradient inversion, the federated learning algorithm FedSGD derived from [14] has been used for all federated learning scenarios, see Algorithm 1. The model construction steps are as follows: 1) The parameter server broadcasts the model parameters to each client; 2) Each client trains the model using part of the local data, and then uploads the updated model gradient to the parameter server, 3) The parameter server aggregates the model gradient of each client to obtain a global model, and broadcasts the parameter of the new global model to each client; then, 4) Steps 2)-3) are repeated for model training till the specified number of iterations is met.

\section{Relationship Between Gradients}

In federated learning, three types of gradients are collected from different sources: 1) Single Gradient: the model gradient of an input sample; 2) Batch Gradient: the model gradient of input samples with a batch size greater than 1;3) Aggregation Gradient: the gradient of the global model aggregated by the parameter server. Under appropriate regularity conditions, we can exchange differentiation and expectation [13], that is, the relationship between the loss function and the parameter gradient is as follows,

$$
\frac{\partial \mathcal{L}}{\partial W}=\frac{\partial \mathbb{E}_{i \sim p}\left[\mathcal{L}_{i}\right]}{\partial W}=\mathbb{E}_{i \sim p}\left[\frac{\partial \mathcal{L}_{i}}{\partial W}\right]
$$

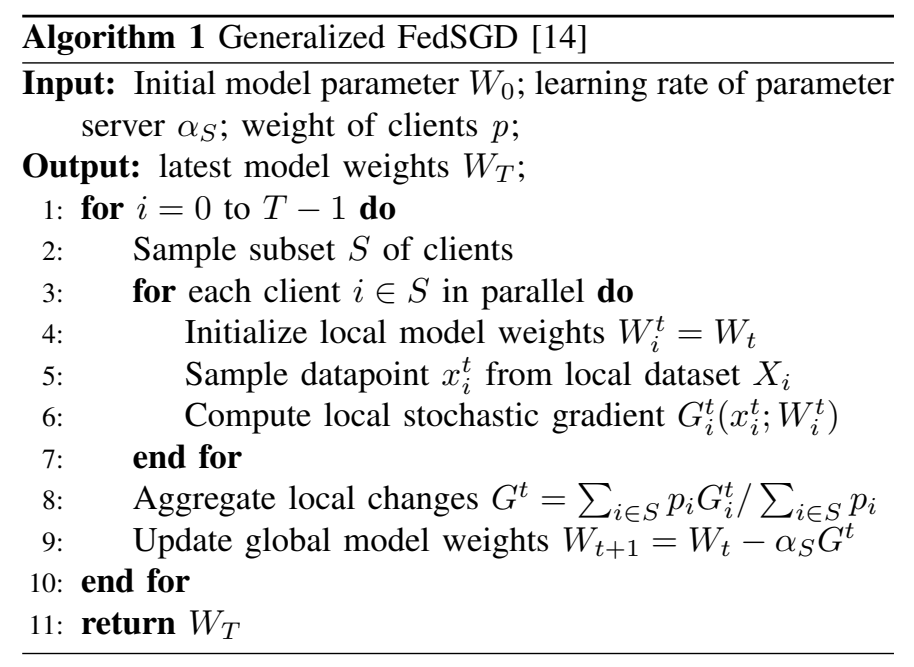

Hence, for the $k^{\text {th }}$ client, its Single Gradient $G^{(i)}$ and Batch Gradient $G_{k}$ have the following conversion relationship:

$$
G^{(i)}=\frac{1}{B_{k}}\left(G^{(1)}+G^{(2)}+\cdots+G^{\left(B_{k}\right)}\right)
$$

where $B_{k}$ is the batch size. The aggregation operation is performed by the parameter server in Algorithm 1: $G^{t}=$ $\sum_{i \in S} p_{i} G_{i}^{t} / \sum_{i \in S} p_{i}$, where the weight of Client $p_{i}$ is the ratio of $B_{k}$ to the total number of samples $B=\sum B_{k}$. Then the Aggregation Gradient for $N$ clients satisfies the following formula:

$$
G=\frac{1}{B} \sum_{k}^{N_{c} \text { lients }} \sum_{j}^{B_{k}} G_{k}^{(j)}
$$

Since the Aggregation Gradient and the Batch Gradient have the above conversion relationship, we will refer to them collectively as the Aggregation Gradient in the rest of the paper.

\section{Who Can Get the Gradients?}

In federated learning, each client uses its own data $\left(x_{i}, y_{i}\right)$ to obtain its local model gradient $G_{i}$. Research on gradient inversion has proved that gradient inversion can be used to reconstruct the data $\left(x_{i}, y_{i}\right)$ from the model gradient of the client. Fig. 2 shows how the input data can be leaked from the gradient. In the case where the data thief is a parameter server, the thief can directly obtain the model gradient of a designated client, and launch a gradient inversion attack against this client. Secure aggregation [15], in which the parameter server executes ciphertext gradient aggregation, can readily defend against this attack. Nevertheless, it will raise the time cost of model construction. The specific situation is not the focus of this paper and will not be discussed. In the case where the data thief is a client, it is impossible to directly obtain the model gradient of a specified client, but by analyzing the changes in the global model parameters, such as formula (5), the thief can obtain the aggregate gradient of all clients in an indirect manner, so that the thief can use gradient inversion to steal the training data of other clients.

$$
G:=\frac{W_{t}-W_{t+1}}{\alpha_{S}}
$$




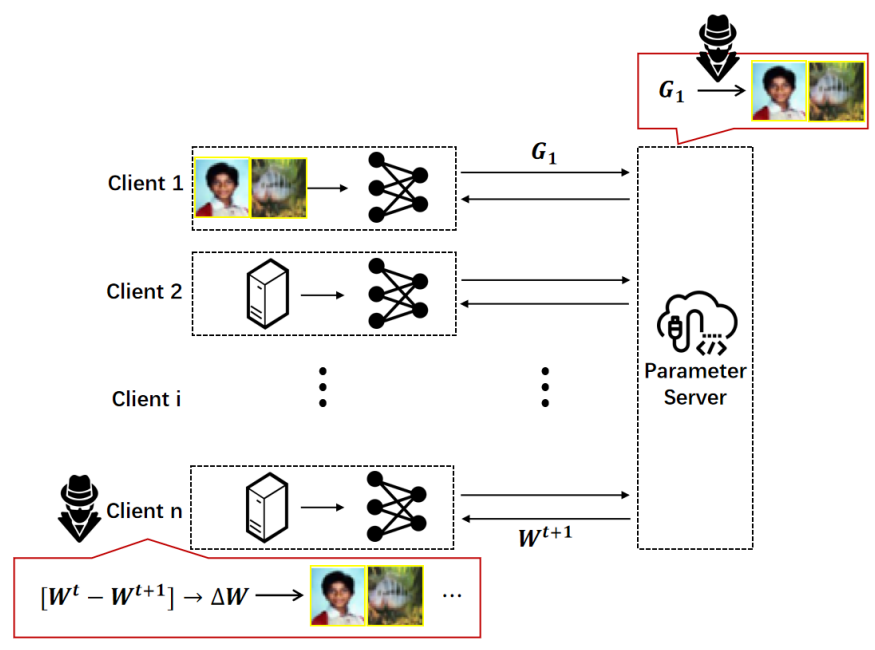

Fig. 2. Schematic diagram of input data leakage from the gradient. The malicious parameter server can directly obtain the gradient uploaded by each client and reconstruct the input sample through gradient inversion; the malicious client can also reconstruct the input sample through gradient inversion by obtaining the parameter changes of the model.

where $W_{t}$ is the global model parameter at the $t^{t h}$ time step, and $\alpha_{S}$ is a constant, which represents the learning rate used in gradient aggregation. The above formula corresponds to the reverse operation of the parameter server to update the parameter in the $9^{\text {th }}$ row of Algorithm 1.

In a word, in a scenario with a parameter server, both the client and the parameter server can obtain the model gradients of other clients to launch gradient inversion attacks and reconstruct training data of other clients.

\section{GRADIENT INVERSION DEFINITION}

\section{A. Hypothesis and Definition}

1) Applicable Scene: The main scene of gradient inversion is cross-silo FL. Kairouz et al. [16] divides the application scenarios of federated learning into cross-device FL and crosssilo FL. Cross-device FL is mainly for scenarios where a large number of mobile devices are jointly modeled, while cross-silo FL is mainly for scenarios where many companies or organizations train a model based on all of their data. In gradient inversion, the attack scenario is more suitable for cross-silo FL, that is, the scenario with a small number of clients and high availability; while the large number of clients and complex client status in cross-device FL makes it rather challenge to carry out gradient inversion attacks.

2) Hypothesis: We use triples $(X, W, G)$ to represent the federated learning clients, where $X$ represents the training sample space of a client, $W$ represents the parameter space of the current global model, and the client needs to upload the model gradient $G[W \times X] \rightarrow \mathbb{R}$. The data thief constructs a sample space $\mathcal{X}$. The hypothesis of effective gradient inversion is that if $G[W \times \mathcal{X}]=G[W \times X]$ is satisfied, then $\mathcal{X}=X$. That is, the data thief can explore a set of samples. Once the model gradient of the set of samples is the same as the model gradient of the target sample, the set of samples is considered to be the same as the target sample.
Based on the above hypothesis, we define the gradient inversion as follows:

Definition 1 (Generalized Gradient Inversion: $g G I$ ): Let $\mathcal{X}$ represent the effective value space of the sample input, $\mathcal{Y}$ represent the effective value space of the sample label, $W$ represent the parameter of the model, and $G$ which is the gradient obtained by the target sample $(x, y)$ to the model parameter $W$ is called the target gradient, $G^{*}$ which is the gradient obtained by the pseudo-sample $\left(x^{*}, y^{*}\right)$ to the model parameter $W$ is called the pseudo-gradient, $\mathcal{D}_{\text {grad }}$ represents the gradient difference between the target sample and the pseudo-sample, $R_{a u x}$ represents the regularization for constraining the $\mathcal{D}_{\text {grad }}$. The optimization problem of a general gradient inversion is described as searching for $\left(x^{*}, y^{*}\right)$ to minimize the distance between the target gradient $G$ and pseudo-gradient $G^{*}$ :

$$
\min _{x^{*} \in \mathcal{X}, y^{*} \in \mathcal{Y}}: \mathcal{D}_{\text {grad }}\left(G, G^{*}\right)+R_{\text {aux }}\left(x^{*}\right)
$$

In the optimization problem of gradient inversion shown in (6), $G$ is an invariant; $G^{*}$ is a variable, which is the dependent variable of the pseudo-input $x^{*}$ and the pseudo-label $y^{*}$. This optimization problem minimizes the gradient difference so that the pseudo sample $\left(x^{*}, y^{*}\right)$ approximates the target sample $(x, y)$.

Fig. 3 shows the schematic of the gradient inversion algorithm, and the generalized gradient inversion algorithm is shown in Algorithm 2. The specific steps are explained as follows: (1) After the $t^{t h}$ round of gradient aggregation of the federated learning model, the data thief first obtains the model gradient of the target client $G$, which is calculated by the target client using local data $(x, y)$; $(2)$ The data thief initializes a batch of pseudo-samples $\left(x^{*}, y^{*}\right)$ with the same number of input samples of the target client, where $x^{*}$ is called pseudoinput, and its corresponding label $y^{*}$ is called a pseudo-label. The gradient $G^{*}$ of the model parameter $W_{t-1}$ is obtained by a pseudo-sample and is called a pseudo-gradient; (3) The difference $\mathcal{D}$ between the pseudo gradient $G^{*}$ and the target gradient $G$ is calculated; (4) The derivative of the gradient difference $\mathcal{D}$ to the pseudo-sample $\left(x^{*}, y^{*}\right)$ is computed to update the pseudo-sample; (5) Steps (3)-(4) are repeated until the specified number of iterations is reached, and the pseudo sample is returned and used as the reconstruction sample.

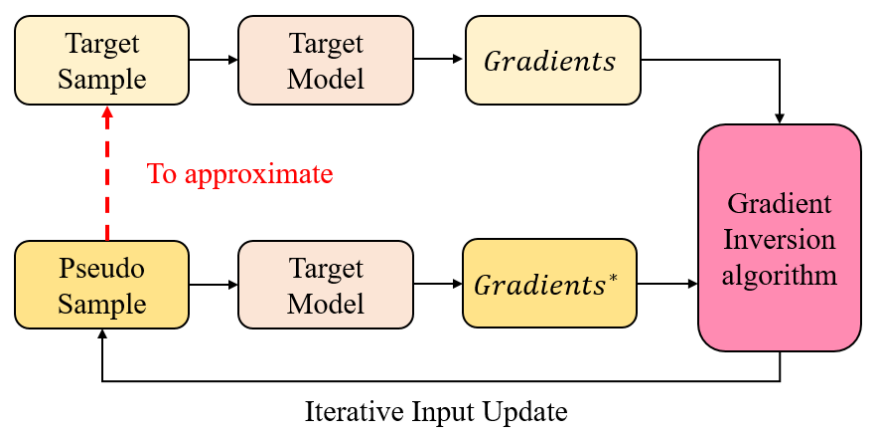

Fig. 3. Schematic diagram of the gradient inversion algorithm. 


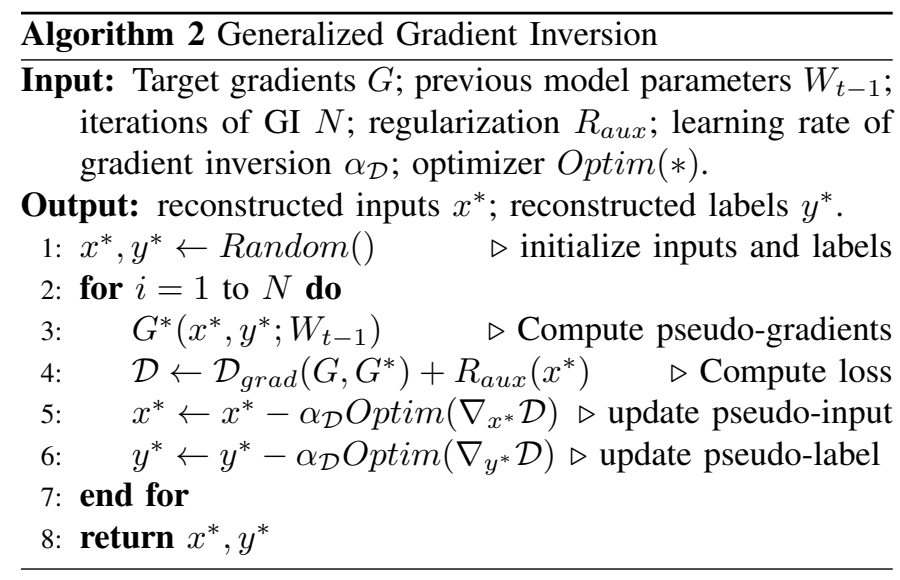

\section{B. The Four Components of Gradient Inversion} Algorithm

The design of gradient inversion algorithm usually involves four parts: optimizer, gradient loss function, label reconstruction method, and regularization, which are also the focus of past works on gradient inversion.

1) Optimizer: The optimization algorithm mainly refers to the algorithm of solving the optimization problem of gradient inversion, as shown in (6). The target sample is reconstructed through a certain optimization strategy, such as the method of gradient descent. Two widely used optimizers for gradient inversion are L-BFGS [17] and Adam [18]. Specific discussions are shown in SectionV-B.

2) Gradient Loss Function: This function is used to measure the difference $\mathcal{D}_{\text {grad }}\left(G, G^{*}\right)$ between the target gradient $G$ and the pseudo-gradient $G^{*}$ constructed by the data thief. Gradients in neural networks are often high-dimensional, so effective methods are required to measure the difference between gradients. The two most intensively-studied gradient difference measurement methods are L2 norm and cosine similarity, which will be discussed in detail in Section V-C.

3) Label Reconstruction Method: This method refers to reconstructing the label of the target sample. In the $6^{\text {th }}$ line of the Generalized Gradient Inversion Algorithm 2, the data thief needs to reconstruct the label of the target sample, and iteratively update the pseudo-label. However, in previous reports [19], [20], the gradient symbol of the fully connected output layer corresponds to the label of the target sample, so the data thief can directly reconstruct the label of the target sample from the target gradient, and the pseudo-label will be fixed and no longer needs to be updated in gradient inversion. In actual experiments, the correctness of label reconstruction plays a very important role in reconstructing samples. Specific discussions are shown in Section V-D.

4) Regularization: The main function of the regularization scheme $R_{a u x}$ is to modify the convergence of the gradient loss function, thereby improving the quality of reconstructed samples. In the gradient inversion process, regularization is required to promote reconstruction of the sample to converge to effective distribution. More details of regularization are shown in Section V-E.

\section{Gradient inVersion deVElopment}

This section focuses on the development history of gradient inversion methods. Using the optimizer as a dividing line, we divide the relevant research into two categories: gradient inversion based on L-BFGS and gradient inversion based on Adam.

\section{A. Gradient Inversion Based on L-BFGS Optimization}

Early works on gradient inversion mainly chose L-BFGS as the optimizer.

1) Deep Leakage from Gradients(DLG): Zhu et al. [8] proposed a method of data leakage from gradients called DLG and its recovery is pixel-wise accurate for images, which laid a basic paradigm for development of gradient inversion methods. DLG uses the L2 norm to measure the gradient loss function $\mathcal{D}_{\text {grad }}\left(G, G^{*}\right)$. Both the input and label in the pseudosample need to be optimized for the gradient loss function. The optimization method is L-BFGS. Using target images from MNIST [21], CIFAR-100 [22], SVHN [23] and LFW [9] datasets, DLG reconstructs the input from the gradient on the ResNet-20 and ResNet-56 models [24], However, Sigmoid is used to replace all of the original ReLU activation functions in the model, and strides are removed because the DLG approach requires the target model to be twice-differentiable. The code of DLG, as published in [25], shows a demonstration code for single sample reconstruction on a four-layer convolutional network. The experimental results for multi-sample reconstruction on the ResNet model are not disclosed.

2) Improved Deep Leakage from Gradients(iDLG): Zhao et al. [19] proposed iDLG on the basis of DLG, and theoretically proved the correspondence between the gradient of the output layer as a fully connected layer and the single sample label. In the current popular convolutional networks, the output layer is often a fully-connected layer. The mechanism of iDLG label reconstruction is that the sign of gradient of the output neuron corresponding to the target label in the fully-connected output layer is opposite to the sign of the gradient of other output neurons. We use $\nabla W_{i}^{[L]}$ to represent the gradient of the $i^{t h}$ neuron of the last layer of the model. In the public code of iDLG [26], the row summation of $\nabla W_{i}^{[L]}$ is $\sum_{k} \nabla W_{i, k}^{[L]}$, whose sign represents the sign of gradient of the $i^{t h}$ output neuron. The target label is inferred by the following formula:

$$
\forall j \neq i, \text { if } \sum_{k} \nabla W_{i, k}^{[L]} \cdot \sum_{k} \nabla W_{j, k}^{[L]}<0 \text {, then } y^{*}=i
$$

In particular, if the activation function used in the hidden layer of the target model is a non-negative function, such as ReLU and Sigmoid, and the sign of $\sum_{k} \nabla W_{i, k}^{[L]}$ is negative, then the target label is $i$. Using the target images from MNIST, CIFAR-100 and LFW datasets, the iDLG method is compared with DLG in terms of the label reconstruction accuracy on the four-layer convolutional network of DLG public code. iDLG achieves a single-sample label reconstruction accuracy of $100 \%$, while DLG on LFW dataset achieves a label reconstruction accuracy at merely $79.1 \%$. Since the iDLG method can infer the target label from the gradient, the label in the pseudo sample does not need to be optimized any more, which greatly improves the quality of the reconstructed sample. 


\section{B. Gradient Inversion Based on Adam Optimization}

In the gradient inversion algorithm DLG based on L-BFGS, the target model is required to be twice-differentiable [8]. When ReLU is used as the activation function of the target model, the gradient inversion algorithm is difficult to converge. However, Adam is not subject to this limitation, so the Adam optimization method is adopted by more subsequent studies.

1) Inverting Gradients(iTG): Geiping et al. [27] proposed iTG on the basis of iDLG. The iTG method differs from iDLG in the following four aspects:

1) In the optimization algorithm, iTG uses Adam to solve the optimization problem of the gradient loss function for the first time. This method can be applied to more mainstream and general models, and the model is no longer required to be twice-differentiable.

2) In the gradient loss function, iTG uses cosine similarity to measure the difference between the target gradient $G$ and the pseudo-gradient $G^{*}$.

$$
\mathcal{D}_{\text {grad }}\left(G, G^{*}\right)=1-\frac{<G, G^{*}>}{\|G\|\left\|G^{*}\right\|}
$$

Geiping et al. [27] believe that the high-dimensional direction of the gradient can carry significant information, as the angle between two data points quantifies the change in prediction at one data point when taking a gradient step towards another [28], [29].

3) In regularization, iTG adopts a total variation $R_{T V}$ [30], [31] for pseudo-input in the gradient loss function, which corresponds to $R_{\text {aux }}$ in (6). The total variation regularization assumes that the value mutation between adjacent pixels is small in a normal image. After the total variation is added, it is beneficial to reduce the noise and improve the quality of the reconstructed image.

$$
R_{T V}=\sum_{i, j}\left(\left(x_{i, j+1}^{*}-x_{i, j}^{*}\right)^{2}+\left(x_{i+1, j}^{*}-x_{i, j}^{*}\right)^{2}\right)^{\frac{1}{2}}
$$

$R_{T V}$ is performed on the pseudo-input $x^{*}$, where $x_{i, j}^{*}$ refers to the pixel value of the $i^{\text {th }}$ row and $j^{\text {th }}$ column of the pseudo-input. It can be seen that lowering the value of $R_{T V}$ can make the image smoother.

4) In terms of the parameter status of the target model, the target model targeted by the iTG method has both untrained parameters and pre-trained ones, and it is verified that the iTG method achieves reconstructed samples of better quality on pre-trained models.

In the public code [32], the iTG method realizes the reconstruction of the input sample from the ImageNet dataset on the pre-trained ResNet-152 model. In addition, the code also shows the iTG method's reconstruction effect when the input sample's batch size is 100, where the images come from the CIFAR-100 dataset, and the target model also uses the ResNet architecture. We can see even if the input sample's batch size is large, the iTG method can still reconstruct part of the input data.

2) Image Batch Recovery via Gradlnversion(BGI): Yin et al. [20] proposed BGI on the basis of iTG, which differs from the latter in the following five aspects:
1) In the gradient loss function, the L2 distance instead of the cosine similarity adopted in iTG is used as gradient loss function in BGI.

2) The addition of the L2 norm regularization of the pseudo sample. BGI adds three regular terms on the gradient loss function, one of which is to penalize the L2 norm of the pseudo sample called $R_{\text {norm }}$. Its function is to limit the size of the L2 norm of the reconstructed image [29], and promote the pseudo sample to converge to an effective value space.

3) The addition of regularization of the BN layer. BGI sets a loss item for the input mean and variance of the BatchNorm layer. The BatchNorm (BN) layer is widely used in deep learning models because it can prevent model overfitting and accelerate model convergence. When performing federated learning of models containing a BN layer, to achieve aggregation of the BN layer, each client needs to upload the input mean and variance of their own $\mathrm{BN}$ layer to the parameter server. The mean and variance of the $\mathrm{BN}$ layer can be utilized by the attacker. For example, the BN layer in the network is the $l+1^{\text {th }}$ layer. For the target client, the input of the $\mathrm{BN}$ layer is $z^{[l]}$, and if its mean mean $\left(z^{[l]}\right)$ and variance $\operatorname{var}\left(z^{[l]}\right)$ are obtained by the data thief after being uploaded to the parameter server, then regularization of the $\mathrm{BN}$ layer can be added to the gradient inversion:

$$
\begin{array}{r}
R_{B N}=\sum_{l}\left\|\operatorname{mean}\left(z^{[l] *}\right)-\operatorname{mean}\left(z^{[l]}\right)\right\| \\
\left.+\sum_{l} \| \operatorname{var}\left(z^{[l] *}\right)-\operatorname{var}\left(z^{[l]}\right)\right) \|
\end{array}
$$

The thief feeds the pseudo-sample $x^{*}$ into the model and gains the input of the BN layer $z^{[l] *}$. The purpose of regularization is to reduce the difference between $z^{[l] *}$ and the target $z^{[l]}$, which speeds up the convergence of the pseudo-sample $x^{*}$ to the target sample $x$, and further improves the gradient inversion effect in the deep network.

4) The addition of group consistency regularization. The initial values of different pseudo samples will be used to obtain reconstructed samples of different quality or expose part of the information of the target sample. For deeper convolutional networks, the reconstructed samples will suffer a certain azimuth misalignment. To merge the reconstructed samples from different orientations to further improve image resolution, BGI uses the image alignment method proposed by Shen et al. [33]. The specific procedure is as follows: (1) First, a number of different pseudo-sample initialization values are used to perform preliminary gradient inversions separately, and then reconstructed samples of different quality are acquired; (2) Average calculation is performed on these reconstructed samples to obtain mean samples; (3) Each reconstructed sample is aligned with the mean sample [33]; (4) The mean operation is performed on the aligned samples to get $\mathbb{E}\left(x_{g \in G}^{*}\right)$; and finally, $\mathbb{E}\left(x_{g \in G}^{*}\right)$ and each reconstructed sample form a group consistency regularization term $R_{\text {group }}$. After a certain number of 
iterations, Steps (2)-(4) will be repeated until certain conditions are met. The formula for group consistency regularization is as follows:

$$
R_{\text {group }}\left(x^{*}, x_{g \in G}^{*}\right)=\left\|x^{*}-\mathbb{E}\left(x_{g \in G}^{*}\right)\right\|
$$

5) BGI has improved the label reconstruction method of iDLG. When the hidden layer of the target model uses non-negative activation functions such as ReLU and Sigmoid, let $B$ denote the number of target samples, and $\nabla W^{[L]}$ the gradient of the last fully-connected layer of the target model, and the formula for BGI to reconstruct the label through the gradient is as follows:

$$
y^{*}=\arg \left(\operatorname{sort}\left(\min _{m} \nabla W_{m, k}^{[L]}\right)[: B]\right)
$$

The iDLG's label reconstruction method uses the sign of the row summation of $\nabla W_{m, k}^{[L]}$ to represent the gradient sign of the the output neuron, while BGI uses the sign of the minimum value $\min _{m} \nabla W_{m, k}^{[L]}$ in each row as the gradient sign of the output neuron. The minimum value is sorted, and the output index corresponding to the smallest $B$ value is selected as the reconstructed label. BGI achieves more fine-grained and robust label reconstruction than iDLG.

The BGI method in [20] can successfully reconstruct images with a batch size 48 from the ImageNet dataset with larger input dimensions on the ResNet-50 model pretrained by MoCoV2 [34], but the code of the BGI method is not disclosed.

\section{ON EVALUATING GRADIENT INVERSION ALGORITHM}

As explained in Section III-B, the components of gradient inversion are mainly composed of optimizer, gradient loss function, label reconstruction method, and regularization. The relevant technologies used in recent works on gradient inversion are listed in Table I. In the present work, experiments are performed to evaluate the role of these components in the gradient inversion algorithm.

\section{A. Experimental Setup}

The current gradient inversion is mainly used for input image reconstruction, so we need to measure the similarity between the reconstructed image and the target image. According to the current image similarity metrics used in relevant literature on gradient inversion, we choose the following four similarity metrics to measure the similarity between images at the pixel level, perception level, and structure level.

1) Mean Square Error (MSE $\downarrow$ ): A measure of similarity at the pixel level. A smaller MSE means a higher similarity;

2) Perceptual Similarity Score (LPIPS $\downarrow$ [35]): A measure of similarity at the perceptual level. A smaller LPIPS means a higher similarity;

3) Peak Signal-to-Noise Ratio (PSNR $\uparrow$ ): Another measure of similarity at the pixel level. A larger PSNR means a higher similarity;
4) Structural Similarity (SSIM $\uparrow$ ): A measure of similarity at the structure level. A larger SSIM indicates a higher similarity.

Fig. 4 shows the changes in the gradient loss and these similarity metrics as the number of iterations increases.

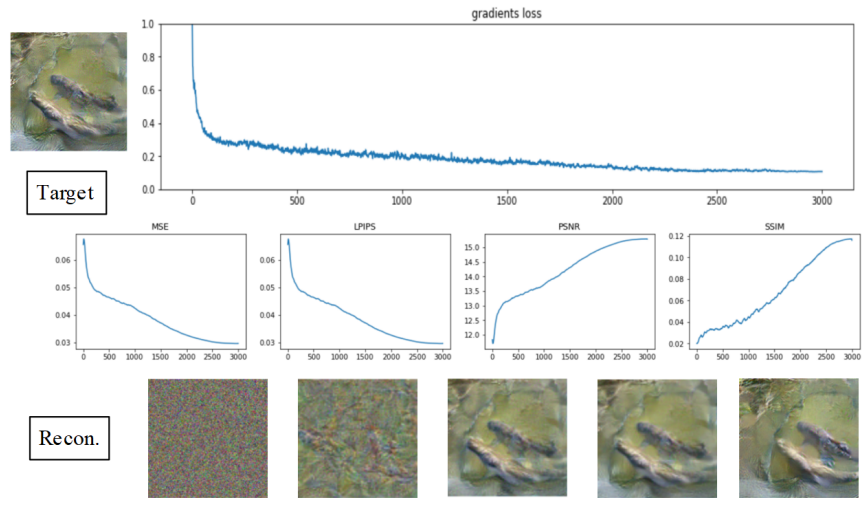

Fig. 4. Change curves of the similarity metrics with decreased gradient loss and the evolution of the reconstructed image. The total number of iterations is $3 \mathrm{~K}$.

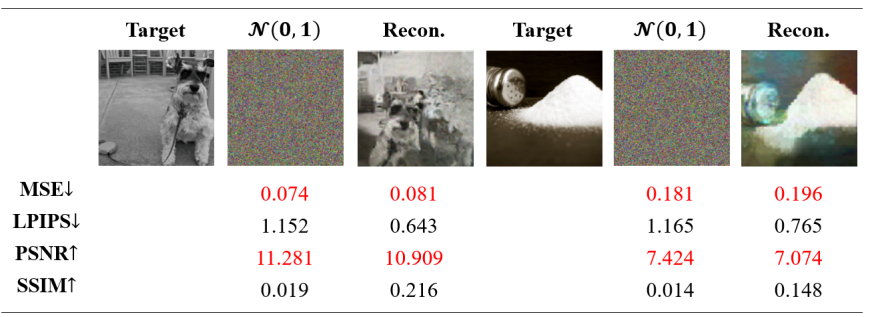

Fig. 5. Four image similarity metrics in gradient inversion.

Through experiments, we found that the metrics MSE and PSNR consider only the differences between pixels, but overlook the semantic similarity of the image, so these metrics become invalid when the objects in the reconstructed image are displaced or the color changes. Fig. 5 shows the measuring results by the four metrics. If we only consider the MSE and PSNR indicators, the similarity between the target sample and the reconstructed image leaking the target sample information may be unexpectedly lower than that between the random image and the target sample. Fortunately, the perceptual similarity score LPIPS and the structural similarity metric SSIM can deal with this problem well. In general, each metric performs well, so we preserve all these four metrics so that the experimental results can be reflected in the similarity at the pixel level, perceptual and structure level.

Experiments are run on a computer with GPU 2080ti. To improve the generalization of the experimental results, we use four models of different scales and parameter states LeNet5 [36], ResNet-18, ResNet-50 pre-trained with MoCoV2; and four datasets of different sizes and types are used as the target sample: grayscale images from MNIST $(28 \times 28)$, color images from CIFAR-100 (32×32), color images from ImageNet $(224 \times 224)$, face images from CelebA $(224 \times 224)$ [37]. The input samples are all standardized by Z-score normalization: new data $=($ original data-mean $) /$ standard deviation, and the 
TABLE I

SUMMARY OF RECENT WORK ON GRADIENT INVERSION

\begin{tabular}{|c|c|c|c|c|}
\hline Method & Optimizer & Gradient Loss Function & Label Reconstruction Method & Regularization \\
\hline DLG [8] & L-BFGS [17] & L2 norm & gGI.(6). & - \\
\hline iDLG [19] & L-BFGS & L2 norm & $\operatorname{sign}\left(\sum_{k} \nabla W_{i, k}^{[L]}\right) .(7)$. & - \\
\hline iTG [27] & Adam [18] & Cosine similarity & Known label & Total variation regularization $R_{T V}$ \\
\hline BGI [20] & Adam & L2 norm & $\operatorname{sort}\left(\min _{m} \nabla W_{m, k}^{[L]}\right) .(12)$. & $\begin{array}{c}\text { Total variation regularization } R_{T V} \text { norm of input regularization } R_{n \text { orm }} \\
\text { BN layer regularization } R_{B N} \\
\text { Group consistency regularization } R_{\text {group }}\end{array}$ \\
\hline
\end{tabular}

parameters of the untrained model are initialized with kaiming uniform [38].

\section{B. Optimizer}

L-BFGS and Adam are the most intensively used optimizers of the gradient loss function in previous works. The iDLG method uses L-BFGS as the optimizer, and the subsequent iTG uses Adam instead. The gradient inversion method using L-BFGS requires that the target model be twice-differentiable, while the current mainstream convolution models mainly use the ReLU activation function, which cannot be twicedifferentiable as required. We compare the results of the iDLG and iTG methods against the public convolution model to reflect the gradient inversion effect of L-BFGS and Adam. The experimental results are shown in Table II. The target samples are selected from the first five training samples of different sizes and types of datasets MNIST $(28 \times 28)$ and CIFAR100 $(32 \times 32)$, corresponding to public convolution models of different scales, i.e., LeNet-5 and ResNet-18. In order to eliminate the influence of model initialization parameters and pseudo-sample initialization values on the gradient inversion effect, 20 sets of pseudo-samples and model parameter initialization values are tested for each sample.

As Table II shows, the gradient inversion based on L-BFGS is relatively weak in the public convolutional model. Adam performs relatively well under different initial values of model parameters and pseudo-sample initial values, and consumes less time, so it is more extensively used in related works.

\section{TABLE II}

COMPARISON OF THE EFFECTS OF THE OPTIMIZED ALGORITHM L-BFGS AND ADAM ON THE GRADIENT INVERSION

\begin{tabular}{c|cc|cc}
\hline & \multicolumn{2}{|c}{ MNIST \& LeNet-5 } & CIFAR-100 \& ResNet-18 \\
Metric & L-BFGS & Adam & L-BFGS & Adam \\
\hline MSE $\downarrow$ & $0.38 \pm 0.17$ & $\mathbf{0 . 0 8} \pm \mathbf{0 . 1 1}$ & $0.12 \pm 0.13$ & $\mathbf{0 . 0 4} \pm \mathbf{0 . 0 4}$ \\
LPIPS $\downarrow$ & $0.41 \pm 0.19$ & $\mathbf{0 . 1 4} \pm \mathbf{0 . 2 1}$ & $0.12 \pm 0.13$ & $\mathbf{0 . 0 5} \pm \mathbf{0 . 0 6}$ \\
PSNR $\uparrow$ & $5.35 \pm 4.23$ & $\mathbf{1 5 . 6 6} \pm \mathbf{6 . 4 7}$ & $15.92 \pm 9.27$ & $\mathbf{1 6 . 4 3} \pm \mathbf{4 . 5 2}$ \\
SSIM $\uparrow$ & $0.09 \pm 0.21$ & $\mathbf{0 . 6 2} \pm \mathbf{0 . 2 5}$ & $0.52 \pm 0.44$ & $\mathbf{0 . 6 1} \pm \mathbf{0 . 3 1}$ \\
Time & $54 \pm 11$ & $\mathbf{1 9} \pm \mathbf{1}$ & $340 \pm 161$ & $\mathbf{2 3 1} \pm \mathbf{1 1 5}$ \\
\hline
\end{tabular}

The target samples are from the MNIST and CIFAR100 datasets, the models are LeNet-5 and ResNet-18, and the metric values are the mean and standard deviation.

\section{Gradient Loss Function}

L2 norm and cosine similarity are the two primary gradient loss functions employed in previous studies. iTG uses the cosine similarity as the gradient loss function, and the following BGI uses L2 norm. To ensure a fair comparison, we chose the pre-trained ResNet-50 with MoCov2 used by Yin et al.'s BGI model [20] in our experiments, and all other variables were kept unchanged to test the impact of the gradient loss function on the gradient inversion effect. The experiment results are shown in Table III. We compared the quality of the reconstructed input when L2 norm and cosine similarity were used as gradient loss functions, and found that the two achieved similar sample reconstruction quality under the same experimental environment.

TABLE III

COMPARE THE EFFECT OF L2 NORM AND COSINE SIMILARITY AS A

\begin{tabular}{c|cccc}
\multicolumn{5}{c}{ GRADIENT LOSS FUNCTION } \\
\hline Method & MSE $\downarrow$ & LPIPS $\downarrow$ & PSNR $\uparrow$ & SSIM $\uparrow$ \\
\hline L2 & $0.07 \pm 0.03$ & $0.57 \pm 0.15$ & $11.79 \pm 1.81$ & $0.3 \pm 0.12$ \\
SIM & $0.08 \pm 0.04$ & $0.58 \pm 0.14$ & $11.45 \pm 2.33$ & $0.26 \pm 0.09$ \\
\hline The metrics are the mean and standard deviation. The target sample batch \\
size is 8. The selected target sample can be seen in 'Target' of 'ImageNet' \\
in Fig.6, and the model is pre-trained ResNet-50 with MoCov2.
\end{tabular}

\section{Label Reconstruction}

The latest label reconstruction method mentioned in the BGI shows strong robustness when there are no repeated labels in the target sample, but it is less applicable to the case of high label repetition. To further prove the above conclusions, we used the dataset CIFAR100 with 100 label types and the dataset CelebA with 10,177 label types to conduct comparative experiments. The model selected was pre-trained ResNet50 with MoCov2 used in the BGI method. We have taken 10,000 random sampling experiments for each dataset. The experiment results are shown in Table IV.

As Table IV shows, when labels of the target samples have repetitions, the accuracy of BGI's label reconstruction decreases as the degree of repetition increases. For the CIFAR100 data set with only 100 label types, as the number of target samples (batch size) increases, the labels become increasingly prone to repetitions, which leads to a larger decrease in the accuracy of label reconstruction. The Relative Acc basically remains above $95 \%$, which indicates that the BGI label reconstruction method has good robustness to nonrepeated labels. Experiments in the Yin et al.'s work [20] have proven that for an ImageNet dataset with 1000 types of labels, when the sample batch size is 96, the label reconstruction accuracy of BGI can reach at least $97.88 \%$, while the label reconstruction method of iDLG can reach only $80.53 \%$, so the BGI label reconstruction method performs better in this 
scenario; for the CelebA data set with 10,177 label types, the label repetition is lower, so the label reconstruction maintains a higher accuracy.

In a word, the BGI label reconstruction method is more effective in scenarios without repetitive target-sample labels than in scenarios with repetitions.

TABLE IV

THE ACCURACY OF BGI LABEL RECONSTRUCTION VARIES WITH THE DEGREE OF REPETITION OF THE TARGET LABEL

\begin{tabular}{c|ccc|ccc}
\hline & \multicolumn{3}{|c}{ CIFAR-100 } & & \multicolumn{3}{c}{$\begin{array}{c}\text { CelebA } \\
\text { BS }\end{array}$} & $\begin{array}{c}\text { Unique } \\
\text { Labels }\end{array}$ & $\begin{array}{c}\text { Relative } \\
\text { Acc }\end{array}$ & $\begin{array}{c}\text { Unique } \\
\text { Acc } \\
\text { Labels }\end{array}$ & $\begin{array}{c}\text { Acc } \\
\text { Accls }\end{array}$ & $\begin{array}{c}\text { Relative } \\
\text { Acc }\end{array}$ \\
\hline $\mathbf{1}$ & $100 \%$ & $100 \%$ & $100 \%$ & $100 \%$ & $100 \%$ & $100 \%$ \\
$\mathbf{8}$ & $96.64 \%$ & $95.51 \%$ & $98.83 \%$ & $99.91 \%$ & $99.89 \%$ & $99.98 \%$ \\
$\mathbf{3 2}$ & $85.24 \%$ & $81.71 \%$ & $95.86 \%$ & $99.53 \%$ & $99.51 \%$ & $99.98 \%$ \\
$\mathbf{6 4}$ & $72.36 \%$ & $69.96 \%$ & $96.68 \%$ & $98.49 \%$ & $98.46 \%$ & $99.97 \%$ \\
\hline
\end{tabular}

The data are CIFAR100 and CelebA, and the corresponding label types are 100 and 10,177, respectively; the model is pre-trained ResNet-50 with MoCov2. Symbols in the table are defined as follows. BS: the batch size of the target sample; Unique Labels: the degree of non-repetition of labels, which is the ratio of the number of target samples with unique labels to the total number of samples; Labels Acc: the accuracy of label reconstruction, which is represented by the ratio of the number of matches between the reconstructed labels and the target labels to the number of samples; Relative Acc: the relative accuracy of label reconstruction, which is the ratio of Labels Acc to Unique Labels, and it is used to indicate the robustness of the BGI method against non-repetition of labels.

\section{E. Regularization}

The gradient loss function regularization used in previous studies is mainly divided into four categories: the first type is the norm of the pseudo sample $R_{\text {norm }}$, which prevents the value of the reconstructed sample from deviating from the effective value space; the second type is total variation regularization $R_{T V}$ for the pseudo sample, which enhances the clarity of the reconstructed picture by noise reduction and smoothing; the third type is the $\mathrm{BN}$ layer mean and variance loss regularization $R_{B N}$, which speeds up the convergence of the reconstructed sample and improves its quality. $R_{B N}$ plays an important role in multi-sample input and gradient inversion of deep networks; the fourth is group consistency regularization $R_{\text {group }}$, which aims to fuse information from multiple reconstructed samples.

In the comparison experiment, we used eight images in the dataset MNIST and ImageNet as the target batch samples, with a batch size of 8 . The corresponding models are the untrained LeNet-5 and the pre-trained ResNet-50 with MoCov2. To obtain better reconstructed samples, we set five sets of pseudo-sample initialization values, and displayed the best experimental results. In our experiments, we found that the regularization scheme $R_{\text {norm }}$ has a small improvement effect, so it is not shown in the comparison experiment. The improvement effect of other regularization schemes on the quality of the reconstructed sample is shown in Table $\mathrm{V}$, and the corresponding effect of the reconstructed sample is shown in Fig. 6. The experiment shows that the total variation regularization schemes $R_{T V}$ and $\mathrm{BN}$ layer mean and variance loss regularization $R_{B N}$ improve the quality of the reconstructed samples more obviously, while the quality of some of the reconstructed samples of $R_{\text {group }}$ is poor. The reason is analyzed in Section VI-B.

TABLE V

COMPARISON OF RECONSTRUCTED SAMPLE QUALITY OF DATASETS MNIST AND IMAGENET WITH THE HELP OF DIFFERENT REGULARIZATION ACCUMULATION

\begin{tabular}{l|cc|cccc}
\hline & \multicolumn{5}{|c}{ MNIST \& LeNet-5 } & \multicolumn{4}{|c}{ ImageNet \& ResNet-50(MoCoV2) } \\
Metric & $\mathcal{D}_{\text {grad }}$ & $+R_{T V}$ & $\mathcal{D}_{\text {grad }}$ & $+R_{T V}$ & $+R_{B N}$ & $+R_{\text {group }}$ \\
\hline MSE $\downarrow$ & 1.32 & $\mathbf{0 . 4}$ & 0.86 & 0.56 & $\mathbf{0 . 5 4}$ & 0.56 \\
LPIPS $\downarrow$ & 2.52 & $\mathbf{0 . 6 4}$ & 5.3 & 4.39 & $\mathbf{3 . 8 7}$ & 4.78 \\
PSNR $\uparrow$ & 64.44 & $\mathbf{1 1 7 . 0 3}$ & 78.43 & 94.33 & $\mathbf{9 8 . 8 1}$ & 92.26 \\
SSIM $\uparrow$ & 2.02 & $\mathbf{6 . 2 2}$ & 0.37 & 2.12 & $\mathbf{2 . 4 5}$ & 1.82 \\
\hline $\mathcal{D}_{\text {grad }}:$ gradient inversion without regularization; $+R_{T V}:$ total variation \\
regularization; $+R_{B N}:$ BN layer input mean and variance loss regularization; \\
$+R_{\text {group }}$ : group consistency regularization. The model corresponding to \\
MNIST is LeNet-5, and the model corresponding to ImageNet is ResNet- \\
50 pre-trained with MoCoV2.
\end{tabular}

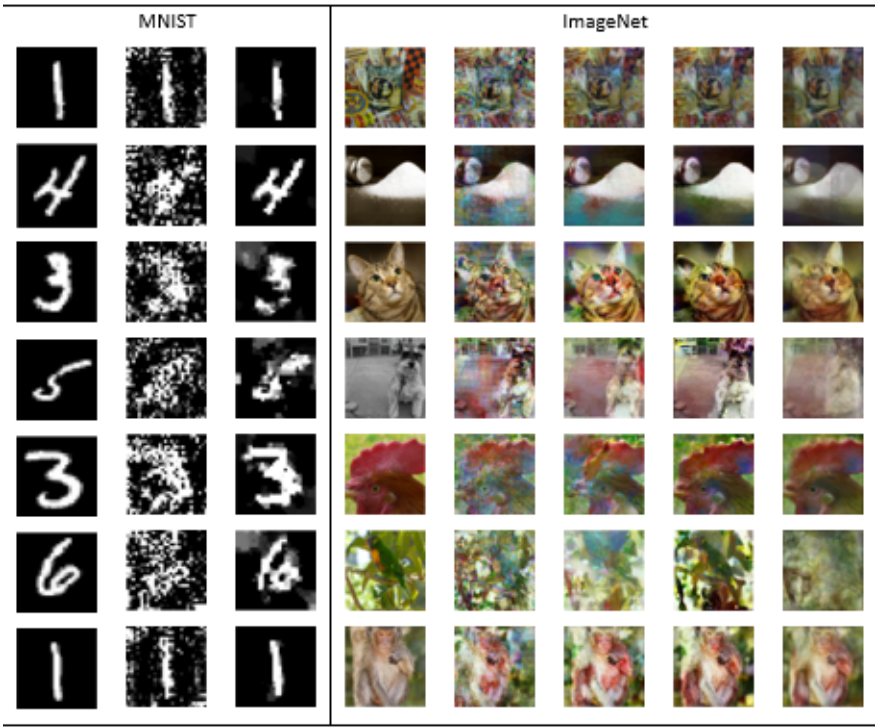

Fig. 6. Comparison image of reconstructed sample quality of dataset MNIST and ImageNet with the help of different regularization accumulation.

\section{OUR FINDINGS}

\section{A. Label Reconstruction Problem and Solution}

BGI's label reconstruction method will inevitably make mistakes on repeated labels because of its own sorting mechanism. The main solution in previous works was to restart the general gradient inversion gGI multiple times by setting different initial values of the pseudo samples to find duplicate labels. However, blind restarts will increase the time cost several times, and in most cases, it is difficult to dig out effective duplicate label clues. The effect is shown in the $2^{\text {th }}, 3^{\text {th }}$ row of Fig. 7 'BGI', 'gGI for $G$ '.

To address above problems, we leverage the conversion relationship between single sample, batch sample gradient and aggregate gradient in (3) (4) to try to provide a solution: when we perform generalized gradient inversion (gGI) on the aggregate gradient, the difficulty of reconstruction is different for different target samples; if we subtract the gradient of the 


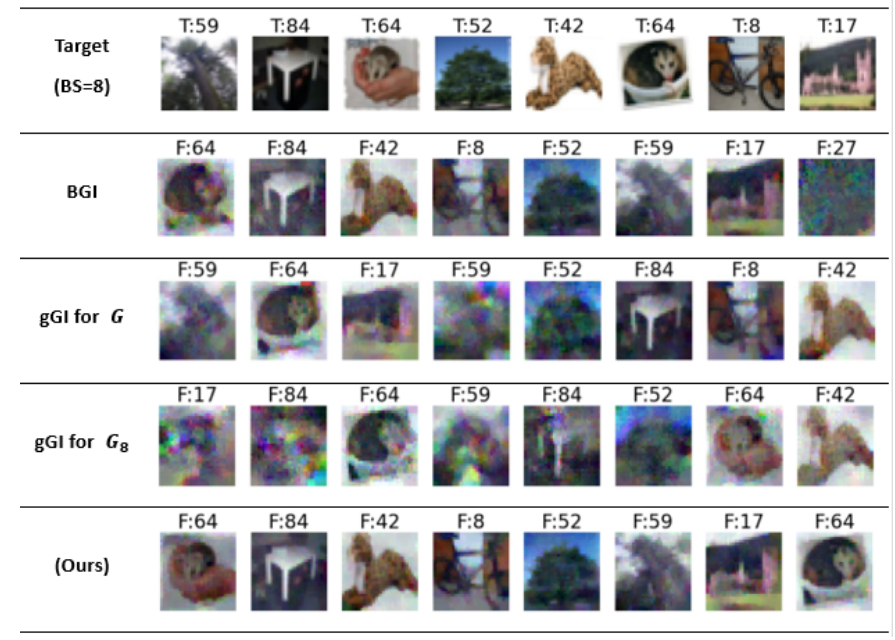

Fig. 7. Comparison of the quality of the reconstructed sample when each label reconstruction method deals with the label duplication of the target sample. The label reconstruction methods BGI, gGI and our proposed method are compared in terms of the quality of reconstructed samples, where $\mathrm{G}$ is the aggregate gradient of the target sample, and $G_{8}$ is the remaining gradient. The target sample comes from CIFAR100, the model is the untrained ResNet-18, and the target sample batch size is 8 .

sample that is easy to be reconstructed from the aggregate gradient to get the remaining gradients, the information of these sample gradients in the aggregated gradient will be reduced. At this time, we apply the gGI approach again on the remaining gradients, and the samples that were not easy to be reconstructed in the past will appear. A specific example is given as follows.

If there are duplicate labels in the target sample, it is difficult to find duplicate labels by the current label reconstruction methods. Specifically, the third label of the target sample in the first row of Fig. 7 is the sample with the label "64", and the label of the sixth sample is also "64", which belongs to the case of duplicate labels. At this time, the BGI method cannot find the third sample clues. Through the above two existing label reconstruction methods, we can determine the unique label $\left([64,84,42,8,52,59,17]\right.$ in the $2_{n d}$ row of Fig. 7 'gGI for $\left.G^{\prime}\right)$. Then, we input the reconstructed samples corresponding to these seven labels into the target model and obtain the corresponding gradient $G_{i}, i=1, \ldots, 7$. We know the aggregate gradient $G$, and calculate $G_{8}=8 * G-\sum_{i=1}^{7} G_{i}$ to obtain the remaining gradient $G_{8}$. Then, we can use the gGI method for the remaining gradient $G_{8}$. Note that the number of pseudo samples must still be the same as the number of original target samples ( $\mathrm{BS}=8$ in this experiment). Because the quality of the reconstructed sample obtained previously is not perfect, the remaining gradient $G_{8}$ still contains a small part of the gradient information of other samples. The result is shown in the $4^{\text {th }}$ row of Fig. 7 ' $\mathbf{g G I}$ for $\boldsymbol{G}_{\mathbf{8}}$ '. We found a new sample and the corresponding label is "64", so it is sure that the label is a duplicate label. Finally, we set the label as known and perform gradient inversion with known labels to achieve reconstruction of all samples, and the quality of reconstructed samples is improved.

\section{B. Group Consistency Regularization Problem}

The application of group consistency regularization entails strict preconditions: most pseudo samples are required to obtain relatively good reconstructed samples; otherwise, it will lead to a poor effect of the group uniform regularization $R_{\text {group }}$, as shown in Fig. 6. The first defect, as demonstrated in Fig. 8, is that the distorted image and azimuth-shifted images easily damage results of group consistency regularization. The second defect is that poor reconstruction quality of some samples directly lead to deterioration of the final result. In general, the final fitting result achieved by group consistency regularization may not be as good as the quality of some initial reconstructed samples. One additional point needs to be explained. If human intervention is introduced when group consistency regularization is employed, intuitively it appears that the final result will not be exceptionally bad because the human eyes can select a better initial gradient inversion result and remove unusually poor initial samples. In fact, we cannot know what the target sample looks like, so it is tough to make a reasonable selection of the reconstructed image obtained.

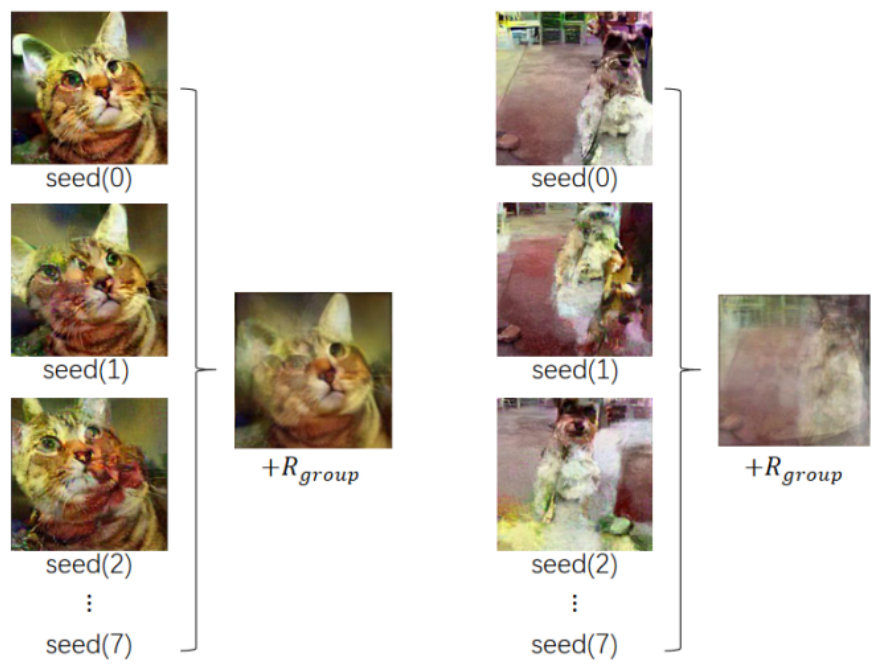

Fig. 8. The effect of group consistency regularization is limited by the results of the initial gradient inversion.

\section{Time Consumption of Gradient Inversion on Pre-trained Model}

In this section, we explore the time consumption of gradient inversion on pre-trained models of different scales. The target samples are three images with different characteristics from the ImageNet dataset. We have labeled 'Simple Multicolor', 'Complex Multicolor' and 'Complex Monochrome', as shown in Fig. 9. We test the average reconstruction sample quality and its time consumption on each pre-trained model of a varied scale. The architecture of the model used is the ResNet convolutional network. The time consumed is the result of an experiment on a single GPU 2080ti. For the gradient inversion algorithm, based on the analysis of regularization in Section $\mathrm{V}-\mathrm{E}$ and the fact that iTG contains the published code, the BGI's BN layer mean and variance loss regularization $R_{B N}$ are incorporated into the algorithm. The number of iterations is 
40K. Table VI shows the experiment result, and Fig. 9 presents the quality of the corresponding partial reconstructed samples. The results reveal that the scale of the pre-training model will not greatly lower the quality of the reconstructed sample, but a deeper model requires more time to reconstruct a image of comparable quality.

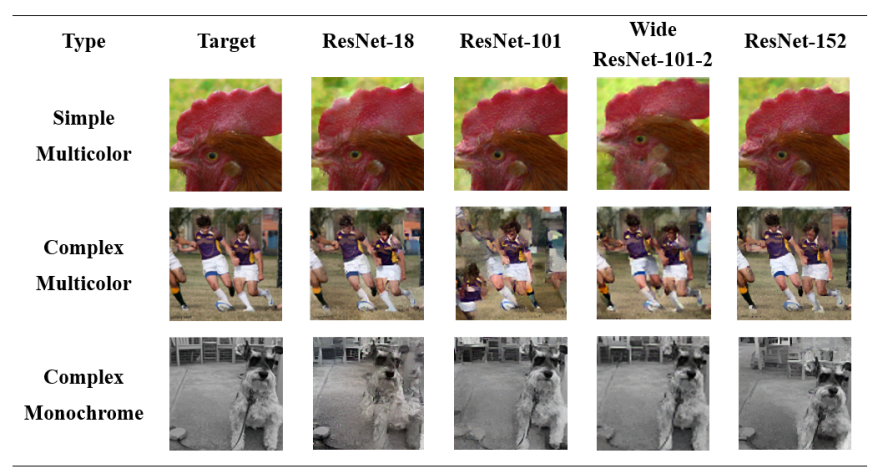

Fig. 9. Comparison of the single-sample gradient inversion effects on different pre-trained models.

TABLE VI

RECONSTRUCTION SAMPLE QUALITY AND TIME CONSUMPTION OF GRADIENT INVERSION OF RESNET MODELS OF DIFFERENT SCALES

\begin{tabular}{c|cccc}
\hline Model & LPIPS $\downarrow$ & PSNR $\uparrow$ & SSIM $\uparrow$ & Time $(\mathrm{s})$ \\
\hline ResNet-18 & $0.29 \pm 0.14$ & $15.83 \pm 3.5$ & $0.37 \pm 0.20$ & 1386 \\
ResNet-34 & $0.27 \pm 0.11$ & $15.42 \pm 2.67$ & $0.32 \pm 0.16$ & 2379 \\
ResNet-50 & $0.4 \pm 0.15$ & $14.25 \pm 3.12$ & $0.31 \pm 0.18$ & 3469 \\
ResNet-50-2 & $0.35 \pm 0.17$ & $14.86 \pm 4.16$ & $0.31 \pm 0.2$ & 3935 \\
ResNet-101 & $0.41 \pm 0.15$ & $13.88 \pm 3.23$ & $0.28 \pm 0.18$ & 7323 \\
ResNet-101-2 & $0.43 \pm 0.13$ & $13.59 \pm 2.29$ & $0.28 \pm 0.16$ & 15351 \\
ResNet-152 & $0.36 \pm 0.16$ & $15.01 \pm 4.38$ & $0.31 \pm 0.20$ & 10968 \\
\hline
\end{tabular}

The batch size is 1 , and metric values are the mean and standard deviation.

\section{Challenges And Opportunities}

\section{A. Challenges of Current Gradient Inversion Algorithms}

In this section, we will discuss the challenges at the gradient inversion algorithm level, as well as its improvement points, and more research points at the application level for gradient inversion

1) Current label reconstruction methods suffer a low accuracy in scenarios where the target samples have a large number of repetitions: As analyzed in Section VI-A, we know that the method of obtaining labels from the gradient of the fully-connected output layer is difficult to deal with repeated labels, and the accuracy of label reconstruction is crucial to reconstruct the input.

2) It is yet to be explored how to better integrate the results of multiple pseudo-samples to improve the final results: Past studies have revealed a phenomenon that the quality of reconstructed samples differs as the corresponding pseudo-samples change, and the reconstructed input details are also different. Yin et al.'s BGI model [20] employs group consistency regularization to fuse the results of multiple pseudo-sample initial values to obtain an outcome. The purpose is to have more input details in the final reconstructed sample. As analyzed in Section VI-B, current methods have plenty of limitations.
3) Gradient inversion consumes too much time on largescale convolutional models: When the target model contains a large number of layers, its corresponding gradient scale is also large, which increases the time cost of gradient inversion accordingly. For example, using GPU 2080ti, 40K rounds of reconstruction on ResNet-152 for a single sample takes more than 3 hours. In order to obtain better image quality, it is also necessary to continuously adjust the hyper-parameter of gradient inversion and restart multiple gradient inversions. For sample batch size greater than 1 , the consumption will be greater.

4) The increased batch size of the target sample will greatly degrade the quality of reconstructed samples: As the batch size grows, the unknown quantity of samples that must be solved for gradient inversion grows as well, which will inevitably increase the difficulty of gradient inversion. The batch size experimented in [20] on the dataset ImageNet with large input dimensions is up to 48 , but the quality of the reconstructed samples has deteriorated dramatically, and even most images are difficult to distinguish. On the dataset CIFAR100 with very small input dimensions, Geiping et al. [27] has experimented with input reconstruction with a batch size 100, and only a minimal part of the images are discernible.

\section{B. Current Research Opportunities for Gradient} Inversion

1) Measuring the difficulty of attacking the model and sample by gradient inversion: Past studies have revealed that the quality of reconstructed samples varies as the model parameters and target samples change; and it is difficult to attack some model parameters and target samples by gradient inversion. Studying the reasons is really beneficial from a defense standpoint.

2) Gradient inversion methods applicable to more data types and more popular model architectures are still to be studied:

At present, research on gradient inversion is mainly concentrated on image data, and the model mainly focuses on fully-connected networks and convolutional networks. In the practical applications of federated learning, structured data are more commonly used as training data, and sequence data such as texts and speeches are getting increasing attention; at the model level, in addition to classification models, regression models are also widely used, and in the financial field, sequence models such as RNN [39], LSTM [40], and the tree model XGBoost [41] have good research value for gradient inversion. For non-image data types and other network architectures, it is particularly important to design differentiated regularization of the gradient loss function to ensure that the reconstructed input can converge to the effective space of its data type.

3) Gradient inversion for more model aggregation methods in federated learning is yet to be studied: Our current research is mainly oriented to the scenario of mean aggregation rule (mean of client gradients), and related literature has recently proposed multiple aggregation rules (e.g., trimmed mean and median [42]). Whether the current gradient inversion is still effective or how to change the algorithm to deal with the new aggregation rule is yet to be studied. 
4) Gradient inversion may amplify the harm of other malicious attacks against federated learning: When a data thief uses gradient inversion to obtain target samples, purposefully modifies the target samples and puts them into model training, it will further enhance the harmfulness of data poisoning, backdoor attacks and other malicious attacks. How should we deal with the new threats?

5) Whether the current defense methods to prevent gradient leakage data are truly effective remains to be discussed: For example, differential privacy sacrifices the usability of the model too much to ensure data privacy [8]. Whether the method of using homomorphic encryption to encrypt gradients and re-aggregation can effectively prevent malicious clients remains to be explored further. As long as the data thief knows the parameter changes between the models, he can receive the updated gradient of the model, and then carry out gradient inversion.

\section{Vili. Related Work}

In addition to gradient inversion, there are other data leakage attacks targeting distributed scenarios. For example, Hitaj et al. [43] proposed a way using a GAN network to steal data in distributed scenarios: the data thief marks the samples generated by the generator as error labels and uses them to train the local model. This makes it difficult for the global model to distinguish the true label of the sample from the wrong one, and the global model will reveal more information about the target data. Wang et al. [44] proposed a gradient inversion method that can target specific clients. The data thief is a parameter server, and the GAN discriminator is used to determine the clients corresponding to the gradient. Finally, you can specify the label and the participant party ID to get the data of the target client generated by the generator; He et al. [45] proposed a data leakage method for collaborative inference. In collaborative inference, the model is split, and each client is responsible for a different part of the model training task. This paper proposes that clients can constantly change the input of the pre-order model to make the corresponding output close to the real output, so as to achieve the purpose of stealing the real input of the pre-order model.

In addition to the data leakage methods specifically proposed for distributed scenarios, there are some other methods.

The model inversion attack was first proposed by $\mathbf{M}$. Fredrikson et al. [46], [47]. Model inversion is to update the random input to minimize the loss between the input classification label and the target label, so as to approximate the inverted input to a training input of the same label [48], [49], [50], such as a person's face. The loss function in the model inversion will add some regularization such as the background information of the target input and the prior information of the face. The latest research is by Zhang et al. [50], who proposed integrating GANs into the model inversion loss function, and using public face datasets to pre-train the GANs network so that the model can construct normal faces, which makes it easier for random input updates to fit real human faces. However, the limitation of model inversion is that it is applicable only to scenarios with similar training data with the same label, such as human faces. It is not suitable for scenarios where the training data with considerably different information like the dataset CIFAR-100 or ImageNet. The introduction of GANs tends to reconstruct samples with similar semantics of the target sample and is difficult to reconstruct target sample at the pixel level.

In addition to the data leakage methods that reconstruct all the information of the target sample, other data leakage methods aim at the property of the target sample, that is, property inference [51], [52], [51]. Ateniese et al. [51] use model parameters as input data and target attributes as labels to train the meta-classifier. Then, with the parameters of the target model input into the meta-classifier, it can be judged whether the target attribute exists, so as to test out the private information of the training data. The goal of other data leakage methods is to determine whether there are specific records in the training data, that is, membership inference [53], [54]. Shokri et al. [53] proposed the idea of membership inference attack for the first time, using a machine learning model to detect the principle that there is a big difference between the prediction vector of specific data and the prediction vector of other data, and by designing an inference model to capture this difference to detect whether there is specific data in the training data.

There are some other richer means of data leakage. Song et al. [55] proposed a data leakage method that can memorize input data through model parameters. Especially for model training platform providers, they can encode the input sensitive information in model parameters during model training. The author also proposes a data stealing method that does not need to modify the training algorithm: this method is similar to data augmentation, which uses some additional synthetic data to expand the original training data set, so that the target model is trained on its expanded data set. In fact, the model is training two different tasks: one is the main task for the original data set, and the other is the malicious task for the synthetic data set. After the model is trained, the data thief only needs to input the specified synthetic data, and the model will output some information of the target training data set. Wei et al. [56] proposed a side-channel attack type of data leakage method. By monitoring the side channel of the CNN accelerator, the attacker can obtain a high-resolution CNN power change trajectory of the accelerator to restore the input information when the $\mathrm{CNN}$ accelerator performs the first layer calculation, so that the attacker can achieve the purpose of data theft. Salem et al. [57] proposed a data leakage method for the black box model of online learning, which proved that the update of the online learning model caused the output change, which would leak the training data of the updated model.

\section{CONCLUSION}

In the first half of this paper, we elaborate on the applicable scenarios, definitions, and components of gradient inversion: gradient inversion is mainly for federated learning scenarios with a small number of clients and high availability. The data thieves are not necessarily the parameter server, and the client can also be the thief. In addition, the gradient of the 
target sample can be acquired indirectly by malicious clients through parameter changes, thereby performing gradient inversion. Then, we sort out the current development context of gradient inversion, and conduct experimental evaluations on the four core technologies of gradient inversion and reveal their existing problems. Finally, this study discusses the research challenges and opportunities of gradient inversion. We hope that this work will help future federated learning privacy protection and advance research in related fields.

\section{REFERENCES}

[1] D. J. Hemanth, V. Rajinikanth, V. S. Rao, S. Mishra, N. M. Hannon, R. Vijayarajan, and S. Arunmozhi, "Image fusion practice to improve the ischemic-stroke-lesion detection for efficient clinical decision making," Evolutionary Intelligence, pp. 1-11, 2021.

[2] B. McMahan, E. Moore, D. Ramage, S. Hampson, and B. A. y Arcas, "Communication-efficient learning of deep networks from decentralized data," in Artificial intelligence and statistics. PMLR, 2017, pp. 12731282.

[3] H. R. Roth, K. Chang, P. Singh, N. Neumark, W. Li, V. Gupta, S. Gupta, L. Qu, A. Ihsani, B. C. Bizzo et al., "Federated learning for breast density classification: A real-world implementation," in Domain Adaptation and Representation Transfer, and Distributed and Collaborative Learning. Springer, 2020, pp. 181-191.

[4] M. J. Sheller, G. A. Reina, B. Edwards, J. Martin, and S. Bakas, "Multiinstitutional deep learning modeling without sharing patient data: A feasibility study on brain tumor segmentation," in International MICCAI Brainlesion Workshop. Springer, 2018, pp. 92-104.

[5] S. Silva, B. A. Gutman, E. Romero, P. M. Thompson, A. Altmann, and M. Lorenzi, "Federated learning in distributed medical databases: Meta-analysis of large-scale subcortical brain data," in 2019 IEEE 16th international symposium on biomedical imaging (ISBI 2019). IEEE, 2019, pp. 270-274.

[6] Z. Yan, J. Wicaksana, Z. Wang, X. Yang, and K.-T. Cheng, "Variationaware federated learning with multi-source decentralized medical image data," IEEE Journal of Biomedical and Health Informatics, 2020.

[7] Triaging covid-19 patients: 20 hospitals in 20 days build ai model that predicts oxygen needs. [Online]. Available: https://blogs.nvidia.com/blog/2020/10/05/federated-learning-covidoxygen-needs.

[8] L. Zhu, Z. Liu, and S. Han, "Deep leakage from gradients," in Advances in Neural Information Processing Systems, H. Wallach, H. Larochelle, A. Beygelzimer, F. d'Alché-Buc, E. Fox, and R. Garnett, Eds., vol. 32. Curran Associates, Inc., 2019.

[9] G. B. Huang, M. Mattar, T. Berg, and E. Learned-Miller, "Labeled faces in the wild: A database forstudying face recognition in unconstrained environments," in Workshop on faces in'Real-Life'Images: detection, alignment, and recognition, 2008.

[10] J. Deng, W. Dong, R. Socher, L.-J. Li, K. Li, and L. Fei-Fei, "Imagenet: A large-scale hierarchical image database," in 2009 IEEE conference on computer vision and pattern recognition. Ieee, 2009, pp. 248-255.

[11] T. S. Brisimi, R. Chen, T. Mela, A. Olshevsky, I. C. Paschalidis, and W. Shi, "Federated learning of predictive models from federated electronic health records," International journal of medical informatics, vol. 112, pp. 59-67, 2018.

[12] W. Li, F. Milletarì, D. Xu, N. Rieke, J. Hancox, W. Zhu, M. Baust, Y. Cheng, S. Ourselin, M. J. Cardoso et al., "Privacy-preserving federated brain tumour segmentation," in International workshop on machine learning in medical imaging. Springer, 2019, pp. 133-141.

[13] J. Wang, Z. Charles, Z. Xu, G. Joshi, H. B. McMahan, M. Al-Shedivat, G. Andrew, S. Avestimehr, K. Daly, D. Data et al., "A field guide to federated optimization," arXiv preprint arXiv:2107.06917, 2021.

[14] J. Chen, X. Pan, R. Monga, S. Bengio, and R. Jozefowicz, "Revisiting distributed synchronous sgd," arXiv preprint arXiv:1604.00981, 2016.

[15] K. Bonawitz, V. Ivanov, B. Kreuter, A. Marcedone, H. B. McMahan, S. Patel, D. Ramage, A. Segal, and K. Seth, "Practical secure aggregation for privacy-preserving machine learning," in proceedings of the 2017 ACM SIGSAC Conference on Computer and Communications Security, 2017, pp. 1175-1191.

[16] P. Kairouz, H. B. McMahan, B. Avent, A. Bellet, M. Bennis, A. N. Bhagoji, K. Bonawitz, Z. Charles, G. Cormode, R. Cummings et al., "Advances and open problems in federated learning," arXiv preprint arXiv:1912.04977, 2019.
[17] D. C. Liu and J. Nocedal, "On the limited memory bfgs method for large scale optimization," Mathematical programming, vol. 45, no. 1, pp. 503-528, 1989.

[18] D. P. Kingma and J. Ba, "Adam: A method for stochastic optimization," arXiv preprint arXiv:1412.6980, 2014.

[19] B. Zhao, K. R. Mopuri, and H. Bilen, "idlg: Improved deep leakage from gradients," arXiv preprint arXiv:2001.02610, 2020.

[20] H. Yin, A. Mallya, A. Vahdat, J. M. Alvarez, J. Kautz, and P. Molchanov, "See through gradients: Image batch recovery via gradinversion," in Proceedings of the IEEE/CVF Conference on Computer Vision and Pattern Recognition, 2021, pp. 16337-16346.

[21] Y. LeCun, "The mnist database of handwritten digits," http://yann. lecun. com/exdb/mnist/, 1998.

[22] A. Krizhevsky, G. Hinton et al., "Learning multiple layers of features from tiny images," 2009.

[23] Y. Netzer, T. Wang, A. Coates, A. Bissacco, B. Wu, and A. Y. Ng, "Reading digits in natural images with unsupervised feature learning," 2011.

[24] K. He, X. Zhang, S. Ren, and J. Sun, "Deep residual learning for image recognition," in Proceedings of the IEEE conference on computer vision and pattern recognition, 2016, pp. 770-778.

[25] dlg. [Online]. Available: https://github.com/mit-han-lab/dlg

[26] Improved-deep-leakage-from-gradients. [Online]. Available: https://github.com/PatrickZH/Improved-Deep-Leakage-from-Gradients

[27] J. Geiping, H. Bauermeister, H. Dröge, and M. Moeller, "Inverting gradients - how easy is it to break privacy in federated learning?" in Advances in Neural Information Processing Systems 33: Annual Conference on Neural Information Processing Systems 2020, NeurIPS 2020, December 6-12, 2020, virtual, 2020.

[28] G. Charpiat, N. Girard, L. Felardos, and Y. Tarabalka, "Input similarity from the neural network perspective," in NeurIPS 2019-33th Annual Conference on Neural Information Processing Systems, 2019.

[29] P. W. Koh and P. Liang, "Understanding black-box predictions via influence functions," in International Conference on Machine Learning. PMLR, 2017, pp. 1885-1894.

[30] L. I. Rudin, S. Osher, and E. Fatemi, "Nonlinear total variation based noise removal algorithms," Physica D: nonlinear phenomena, vol. 60, no. 1-4, pp. 259-268, 1992.

[31] A. Mahendran and A. Vedaldi, "Understanding deep image representations by inverting them," in Proceedings of the IEEE conference on computer vision and pattern recognition, 2015, pp. 5188-5196.

[32] invertinggradients. [Online]. https://github.com/JonasGeiping/invertinggradients.

[33] X. Shen, F. Darmon, A. A. Efros, and M. Aubry, "Ransac-flow: generic two-stage image alignment," in Computer Vision-ECCV 2020: 16th European Conference, Glasgow, UK, August 23-28, 2020, Proceedings, Part IV 16. Springer, 2020, pp. 618-637.

[34] K. He, H. Fan, Y. Wu, S. Xie, and R. Girshick, "Momentum contrast for unsupervised visual representation learning," in Proceedings of the IEEE/CVF Conference on Computer Vision and Pattern Recognition, 2020, pp. 9729-9738.

[35] R. Zhang, P. Isola, A. A. Efros, E. Shechtman, and O. Wang, "The unreasonable effectiveness of deep features as a perceptual metric," in Proceedings of the IEEE conference on computer vision and pattern recognition, 2018, pp. 586-595.

[36] Y. LeCun, L. Bottou, Y. Bengio, and P. Haffner, "Gradient-based learning applied to document recognition," Proceedings of the IEEE, vol. 86, no. 11, pp. 2278-2324, 1998.

[37] Z. Liu, P. Luo, X. Wang, and X. Tang, "Deep learning face attributes in the wild," in Proceedings of the IEEE international conference on computer vision, 2015, pp. 3730-3738.

[38] K. He, X. Zhang, S. Ren, and J. Sun, "Delving deep into rectifiers: Surpassing human-level performance on imagenet classification," in Proceedings of the IEEE international conference on computer vision, 2015, pp. 1026-1034.

[39] W. Zaremba, I. Sutskever, and O. Vinyals, "Recurrent neural network regularization," arXiv preprint arXiv:1409.2329, 2014.

[40] S. Xingjian, Z. Chen, H. Wang, D.-Y. Yeung, W.-K. Wong, and W.c. Woo, "Convolutional lstm network: A machine learning approach for precipitation nowcasting," in Advances in neural information processing systems, 2015, pp. 802-810.

[41] T. Chen and C. Guestrin, "Xgboost: A scalable tree boosting system," in Proceedings of the 22nd acm sigkdd international conference on knowledge discovery and data mining, 2016, pp. 785-794.

[42] D. Yin, Y. Chen, R. Kannan, and P. Bartlett, "Byzantine-robust distributed learning: Towards optimal statistical rates," in International Conference on Machine Learning. PMLR, 2018, pp. 5650-5659. 
[43] B. Hitaj, G. Ateniese, and F. Perez-Cruz, "Deep models under the gan: information leakage from collaborative deep learning," in Proceedings of the 2017 ACM SIGSAC Conference on Computer and Communications Security, 2017, pp. 603-618.

[44] Z. Wang, M. Song, Z. Zhang, Y. Song, Q. Wang, and H. Qi, "Beyond inferring class representatives: User-level privacy leakage from federated learning," in IEEE INFOCOM 2019-IEEE Conference on Computer Communications. IEEE, 2019, pp. 2512-2520.

[45] Z. He, T. Zhang, and R. B. Lee, "Model inversion attacks against collaborative inference," in Proceedings of the 35th Annual Computer Security Applications Conference, 2019, pp. 148-162.

[46] M. Fredrikson, E. Lantz, S. Jha, S. Lin, D. Page, and T. Ristenpart, "Privacy in pharmacogenetics: An end-to-end case study of personalized warfarin dosing," in 23rd \{USENIX\} Security Symposium ( $\{$ USENIX\} Security 14), 2014, pp. 17-32.

[47] M. Fredrikson, S. Jha, and T. Ristenpart, "Model inversion attacks that exploit confidence information and basic countermeasures," in Proceedings of the 22nd ACM SIGSAC conference on computer and communications security, 2015, pp. 1322-1333.

[48] X. Wu, M. Fredrikson, S. Jha, and J. F. Naughton, "A methodology for formalizing model-inversion attacks," in 2016 IEEE 29th Computer Security Foundations Symposium (CSF). IEEE, 2016, pp. 355-370.

[49] S. Yeom, I. Giacomelli, M. Fredrikson, and S. Jha, "Privacy risk in machine learning: Analyzing the connection to overfitting," in 2018 IEEE 31st Computer Security Foundations Symposium (CSF). IEEE, 2018, pp. 268-282.

[50] Y. Zhang, R. Jia, H. Pei, W. Wang, B. Li, and D. Song, "The secret revealer: Generative model-inversion attacks against deep neural networks," in Proceedings of the IEEE/CVF Conference on Computer Vision and Pattern Recognition, 2020, pp. 253-261.

[51] G. Ateniese, L. V. Mancini, A. Spognardi, A. Villani, D. Vitali, and G. Felici, "Hacking smart machines with smarter ones: How to extract meaningful data from machine learning classifiers," International Journal of Security and Networks, vol. 10, no. 3, pp. 137-150, 2015.

[52] L. Melis, C. Song, E. De Cristofaro, and V. Shmatikov, "Exploiting unintended feature leakage in collaborative learning," in 2019 IEEE Symposium on Security and Privacy (SP). IEEE, 2019, pp. 691-706.

[53] R. Shokri and V. Shmatikov, "Privacy-preserving deep learning," in Proceedings of the 22nd ACM SIGSAC conference on computer and communications security, 2015, pp. 1310-1321.

[54] M. Backes, P. Berrang, M. Humbert, and P. Manoharan, "Membership privacy in microrna-based studies," in Proceedings of the 2016 ACM SIGSAC Conference on Computer and Communications Security, 2016, pp. 319-330.

[55] C. Song, T. Ristenpart, and V. Shmatikov, "Machine learning models that remember too much," in Proceedings of the 2017 ACM SIGSAC Conference on computer and communications security, 2017, pp. 587601.

[56] L. Wei, B. Luo, Y. Li, Y. Liu, and Q. Xu, "I know what you see: Power side-channel attack on convolutional neural network accelerators," in Proceedings of the 34th Annual Computer Security Applications Conference, 2018, pp. 393-406.

[57] A. Salem, A. Bhattacharya, M. Backes, M. Fritz, and Y. Zhang, "Updates-leak: Data set inference and reconstruction attacks in online learning," in 29th \{USENIX\} Security Symposium ( $\{$ USENIX\} Security 20), 2020, pp. 1291-1308. 